\title{
Designing for Innovation: Using Enterprise Ontology Theory to Improve Business-IT Alignment
}

\author{
Philip Huysmans, Kris Ven, and Jan Verelst \\ University of Antwerp, Belgium \\ $\{$ philip.huysmans, kris.ven, jan.verelst $\}$ @ua.ac.be
}

In today's economy, innovation plays an increasingly important role in the strategy of organizations. Managers therefore need to understand and be able to manage the innovation process. It has been noted that "[a]t a time when so much attention is given to innovation and entrepreneurship, it is rather pathetic that a deep understanding of the process is lacking. It is no wonder that firms and governments have difficulty trying to stimulate (and manage) innovation when its fundamental processes are so poorly understood." [2, p. 3].

In our research, we aim to contribute to a more effective innovation process by focusing on the structure of organizational artefacts which allows the realization of innovations. This research direction is framed in the emerging scientific area called Enterprise Engineering. Enterprise Engineering builds on existing approaches including organizational theory and information systems sciences in order to purposefully design organizations. The ability to adopt innovations depends largely on the ability to realize changes to the organizational artefacts. There is consensus in literature that information technology (IT) is an important enabler for the implementation of innovations. Most enterprise architecture frameworks acknowledge the importance of aligning the information technology (IT) infrastructure with the enterprise architecture. Therefore, the recent research efforts in the enterprise architecture domain are very relevant for innovation research in enterprise engineering.

This paper, which was originally presented at the ICITIE conference [1], contributes to insights regarding three important issues in the enterprise architecture domain. First, organizations are competing in increasingly volatile environments. In such environments, no long-term competitive advantages can be obtained, and organizations need to strive towards realizing a succession of short-term competitive advantages. Therefore, the enterprise and its supporting IT architecture should be well-aligned, in order to be able to quickly adapt to changing environments.

Second, many enterprise architecture frameworks have a descriptive, rather than prescriptive nature. Although such frameworks are able to describe the original and the revised structure of the organization, it remains unclear why the applied changes resulted in a desirable outcome for the organization. This insight is essential to be able to repeat the architectural process in the future. Consequently, using these frameworks does not contribute to the understanding of the innovation process. 
Third, it has been observed that enterprise architecture frameworks constitute a heterogeneous collection without clear integration, and that their theoretical foundations are currently still limited. A common theoretical foundation at the organizational and information systems level could improve alignment, and could provide prescriptive rules for the architecture. In our research, we propose modularity as a theoretical foundation. Modularity has already been used at both the organizational and information systems level.

As argumented by Enterprise Engineering, a systematic and scientific approach for constructing enterprise architectures should contribute to a solution for these three issues. Therefore, we analyze in this paper a case study of a successful enterprise architecture project with these issues in mind. In the case study, we focus on a European organization that is able to realize substantial improvements in implementing innovations by aligning its IT and enterprise architecture. In both architectures, the same modular structure is used to create loosely coupled entities. At the IT level, the application portfolio consists of loosely coupled applications which are based on high-level, stable business activities from the enterprise architecture. This approach allows business and IT staff to implement changes more quickly, whereas IT used to be considered as a bottleneck in innovation projects. More specifically, we identify advantages in the areas of alignment, change assessment, reuse, and improvements to the development process. Notwithstanding the successful outcome of this enterprise architecture project, the approach taken by the organization strongly relies on the heuristic knowledge of employees: no guidelines or principles are used to identify the appropriate granularity of the modules. As a result, the repeatability and reproducibility of their approach is limited, and no explicit knowledge about the alignment is gained. In order to develop a better understanding of the process elements which can lead to the identified results, a more systematic method is proposed.

Based on the insights from practice, we therefore attempt to contribute to a more systematic method to construct enterprise architectures. We take a design science approach by repeating the enterprise architecture project using the Enterprise Ontology theory. Our results show that the model created by following the Enterprise Ontology theory was very similar to the model created by the organization, which is a desirable result. The main advantage of Enterprise Ontology is that it provides a more repeatable and reproducible result and that the resulting models are more evolvable. This result shows that scientific research in Enterprise Engineering can be relevant and applicable.

\section{References}

1. Huysmans, P., Ven, K., Verelst, J.: Designing for innovation: Using enterprise ontology theory to improve business-it alignment. In: Shishkov, B., Tsihrintzis, G.A., Virvou, M. (eds.) Proceedings of the 1st International Conference on IT-enabled Innovation in Enterprise (ICITIE 2010). pp. 177-186. SciTePress (July 2010)

2. Teece, D.J. (ed.): The Competitive Challenge: Strategies for Industrial Innovation and Renewal. Ballinger Publishing Company, Cambridge, MA (1987) 